\title{
Novel and Emerging Capabilities that Can Provide a Holistic Understanding of the Plant Root Microbiome
}

\author{
Esther Singer, ${ }^{1,2, \dagger}$ John P. Vogel, ${ }^{1,2,3}$ Trent Northen, ${ }^{1,2}$ Christopher J. Mungall, ${ }^{2}$ and Thomas E. Juenger ${ }^{4}$ \\ 1 Joint Genome Institute, 1 Cyclotron Road, Berkeley, CA 94720 \\ ${ }^{2}$ Lawrence Berkeley National Laboratory, 1 Cyclotron Road, Berkeley, CA 94720 \\ ${ }^{3}$ Department of Plant and Microbial Biology, University of California, Berkeley, 111 Koshland Hall, Berkeley, CA 94720 \\ ${ }^{4}$ The University of Texas at Austin, 2415 Speedway, Austin, TX 78712
}

Accepted for publication 3 November 2020.

\section{ABSTRACT}

In recent years, the root microbiome (i.e., microorganisms growing inside, on, or in close proximity to plant roots) has been shown to play an important role in plant health and productivity. Despite its importance, the root microbiome is challenging to study because of its complexity, heterogeneity, and subterranean location. Fortunately, root microbiome research has seen a tremendous influx of novel technologies (e.g., imaging tools, robotics, and molecular analyses), experimental platforms (e.g., micro- and mesocosms), and data integration, modeling, and prediction tools in the past decade that have greatly increased our ability to dissect the complex network of interactions between above- and belowground environmental parameters, plants, bacteria, and fungi that dictate soil and broader ecosystem health. Herein, we discuss methods that are currently used in root microbiome research and that can be expanded to phytobiome research in general ranging from laboratory studies to mesocosm-scale studies and, finally, to field studies; evaluate their relevance to ecosystem studies; and discuss future root microbiome research directions.

Keywords: agriculture, ecology, metagenomics, microbiome, mycology, nutrient cycling, plants, rhizosphere and phyllosphere, soil ecology, soils
Root microbiota associate with every land plant and show community compositions and dynamics that are distinct from the surrounding soil microbial community (Lundberg et al. 2012). Both rhizosphere (the immediate area around the roots) and root endosphere (within the root) microbiomes affect plant health and soil health via processes such as mineral and nutrient turnover (Alegria Terrazas et al. 2016; Wei et al. 2019) and pathogen suppression (Carrión et al. 2019). Attribution of specific processes to distinct microbial players or populations is challenging because soil ecosystems are among the most complex environments on Earth (Fierer

${ }^{\dagger}$ Corresponding author: E. Singer; esinger@lbl.gov

Funding: This work was supported by the U.S. Department of Energy (DOE) Joint Genome Institute, a DOE Office of Science User Facility, and was further supported by the Office of Science (BER), U.S. DOE, Office of Science, Office of Biological and Environmental Research, Genomic Science Program grant number DE-SC 0014156 (to T. E. Juenger) and the Trial Ecosystem Advancement for Microbiome Science and the Microbial Community Analysis and Functional Evaluation in Soils Programs at Lawrence Berkeley National Laboratory award DE-AC02-05CH11231 (to T. Northen); and under the LBNLLDRD entitled "Improving biofuel crop yield from field to lab under drought stress (to E. Singer).

The author(s) declare no conflict of interest.

This article is in the public domain and not copyrightable. It may be freely reprinted with customary crediting of the source. The American Phytopathological Society, 2021.
2017). Soils are made up of a multitude of heterogeneous abiotic and biotic components that interact in a dynamic fashion over a range of spatial and temporal scales (Fig. 1). Soils are categorized based on their physical structure and their mineralogical composition. Soil type, together with climatic characteristics, allows for the development and activity of biological constituents that are specific to a given soil in a particular location and can vary dramatically among soils and locations (Fierer 2017). Those biological constituents can include plants, insects, bacteria, archaea, and fungi, which all contribute to and feed off of the biogeochemical cycles in a given soil. The resulting complex network of interactions is extremely challenging to disentangle due to technological limitations and insufficient information in biological and chemical reference databases (Fierer 2017).

Furthermore, soils contain a vast diversity of microorganisms, which are heterogeneously distributed and engage in frequent horizontal gene transfer. Despite this, most root microbiome studies present data from single time points or single locations and primarily conduct amplicon sequencing combined with limited information on plant or environment. Although the average values provided by such studies may suggest some interactions or mechanisms, few studies follow up with the comprehensive sampling necessary to definitively understand these mechanisms and interactions. In addition, single-point studies are difficult to compare or extrapolate to other environments or plants because 
measured values can vary dramatically over time (e.g., some bacteria bloom early in a growing season only to nearly disappear as plants reach maturity) (Edwards et al. 2018).

Soil and other environmental characteristics can be important indicators of biogeochemical processes that have occurred in the past or are ongoing. Generally, few root and soil microbiome studies take advantage of the relatively inexpensive techniques (e.g., in situ soil sensors, determination of soil moisture via weightper-volume measurements, and $\mathrm{pH}$ strips) to measure soil characteristics. Data on parameters such as $\mathrm{pH}$, volumetric water content, temperature, and salt concentration could allow researchers to draw correlations between microbial activity, plant productivity, and environmental parameters and facilitate opportunities to crossreference studies conducted under comparable conditions.

In the last decade, the root microbiome research community has made tremendous progress in understanding the complexity of soil ecosystems through improvements in experimental methods at both laboratory and field scales. These exciting technological and scientific advancements pave the way forward in root microbiome research. This review summarizes recent technological advancements and the resulting research opportunities categorized by ecosystem component and scale (abiotic, biotic, space, and time), and ends with an outlook and potential applications for phytobiome research.

\section{IMAGING TOOLS}

Root structural imaging. Microbial colonization of the root and rhizosphere can significantly affect root phenology and metabolism. Roots demonstrate enormous phenotypic plasticity with respect to anatomy, shape, cell type, cellular structure, metabolism, and biochemical composition, and these characteristics contribute tremendously to root exudation variation and, as a result, to microbial community differentiation (Saleem et al. 2018). These reciprocal interactions between roots and microbes are not well understood but their direct link showcases the fact that, for understanding root microbiomes, a foundational understanding of root biology is required.

Although hyperspectral imaging of leaves has been broadly applied to monitor plant health, even simple imaging of intact roots has lagged behind due to the challenges presented by the opaqueness of soil (Bodner et al. 2017). Ideally, imaging of root architecture, microbes, and chemical composition as well as visualization of fluxes such as carbon flow through plant compartments and into the soil would be conducted at multiple temporal and spatial scales. Most current methods for analyzing root growth either require artificial growing conditions (e.g., hydroponics and gels), are severely restricted in the fraction of roots detectable (e.g., rhizotrons), or are destructive (e.g., soil coring). For example, many root phenotypic datasets have employed coring or "shovelomics", subsequent root picking and washing, and imaging using light imagers such as the RhizoVision Crown platform (Mattupalli et al. 2019). This method provides valuable information about root architecture; however, it is extremely laborious, it is often not feasible to excavate deep roots, it can remain unknown how much of the root system was recovered and scanned, and root excavation often times terminates the experiment for the selected plants. All of these methods are severely limited because they are destructive, low throughput, or artificial. The later point is particularly important because root architecture can be significantly affected by plant genetics, environmental conditions, soil type, and rootcolonizing bacteria and fungi (Gamalero et al. 2004; Mantelin et al. 2006).

Magnetic resonance imaging (MRI) presents a noninvasive modality that addresses some of the limitations of other root measurement techniques. When coupled with an analysis pipeline in an automated system, MRI can monitor root mass, length, diameter, tip number, growth angles (in two-dimensional polar coordinates), and spatial distribution in a high-throughput manner (van Dusschoten et al. 2016). Similarly, X-ray computed tomography (CT) scanning can provide a comprehensive picture of root systems as long as the roots have a diameter larger than the instruments' resolution (approximately $0.5 \mathrm{~mm}$ for medical CT scanners) (Lin and Alessio 2009). Hence, small plants or young roots are not likely to be resolved well. Another limitation common to both MRI and CT technology is that plants must be grown in pots that fit into the imaging machines and the applicability of MRI and $\mathrm{X}$-ray CT in three-dimensional (3D) imaging of root systems across various pot sizes was recently evaluated (Metzner et al. 2015). Although both MRI and CT were able to resolve high-quality 3D images of root systems in vivo, the reconstructed length and image details differed significantly between the two methods. In small pots, CT outperformed MRI and provided more details thanks to higher resolution whereas, in large pots, MRI was able to display root systems more comprehensively than CT. Soil features such as minerals and burrows can be resolved with CT, while MRI can measure water content in roots and soil. Both CT and MRI, struggled with roots thinner than $400 \mu \mathrm{m}$ (Metzner et al. 2015).

Root vasculature imaging. There are a number of imaging tools that can resolve root vasculature. Using Synchrotron X-ray microtomography, Milien et al. (2012) contrasted the 3D images of vascular systems of successful and unsuccessful graft interfaces in vine rootstocks. Others have applied synchrotron X-ray microtomography to visualize drought-induced embolism in various plant species (Brodersen et al. 2013; Cochard et al. 2015; Torres-Ruiz et al. 2015; Voltolini et al. 2017), to correlate root hair with rhizosphere soil structure formation (Koebernick et al. 2017), and to quantify root-induced changes of rhizosphere physical properties (Aravena et al. 2013). Although synchrotron X-ray micro-CT can render unprecedented detail into the microanatomy of plants and microorganisms, the focus window is relatively limited ( 1 to $2 \mathrm{~cm}$ ) and biological samples tend to lose viability as a result of the intense X-ray radiation.

There are various other imaging methods that have been recently developed or applied to phytobiome research, including superresolution confocal imaging, which can enhance 3D mapping of root and microbial or fungal cells and showcase green fluorescent proteins (Glaeser et al. 2016), and correlative confocal and focused ion beam tool with integrated scanning electron microscope, which allows for extremely fine-scaled 3D mapping (Lucas et al. 2014). When applied individually or in combination, the abovementioned imaging methods will provide opportunities to visualize plant tissue and attached or internally residing bacteria, fungi, and viruses at unprecedented resolution, as well provide information about their physical and chemical context. Because root development is vital for plant health, expansion of root image databases and novel correlations between above- and belowground plant features will enhance our understanding of plant response to environmental and biological stimuli.

\section{UNMANNED AERIAL VEHICLES}

An important goal of the plant-microbiome field is to discover beneficial or deleterious effects of microbes. This means that recording and understanding plant phenotypes and linking them to microbiome variation is key. Similarly, plant microbiomes are intimately tied to the background soil; hence, monitoring soil characteristics is important but can be challenging and laborintensive at appropriate temporal or spatial scales. 
Unmanned aerial vehicles (UAVs) (e.g., "fixed wing" and rotocopters) equipped with RGB cameras, infrared (IR) cameras, multispectral and hyperspectral cameras, GPS, navigation systems, programmable controllers, and automated flight planning have emerged as powerful tools for nondestructive, high-throughput field phenotyping that can be performed throughout the growth season (Liebisch 2015). This has removed a bottleneck in phenotyping but automated processing of this data still presents various challenges, which are discussed elsewhere (Minervini et al. 2015). Monitoring of agricultural fields using drones has become popular among researchers (as well as agronomists, agricultural engineers, and farmers) to more accurately plan and manage their experimental operations. Drones can produce precise maps of soil characteristics and plant characteristics (Bendig et al. 2014), as well as determine irrigation needs, nitrogen levels, and pest occurrence (Huuskonen and Oksanen 2018; Iost Filho et al. 2020). RGB, IR, and hyper- as well as multispectral cameras attached to drones can collect images of the aboveground portion in a range of wavelengths. The resulting data can produce, for example, a vegetation index describing the amount of wavelengths of light emitted from a crop and, hence, can trigger irrigation systems or evaluate the sensitivity of crop breeds to soil moisture in a high-throughput manner (Virlet et al. 2015). Image data can also provide information about plant health status over time and in dependence on the field location and, thereby, allows the employment of an early warning and response system to plant disease or stress (Mahlein 2016).

Traditionally, measuring soil quality parameters (e.g., nitrogen/ phosphorus/potassium ratios or organic matter content) requires destructive sampling and laboratory analyses that are laborious, slow, or expensive. Similarly, root phenotyping requires time- and laborintensive processing and scanning of root tissue to collect data such as root length density and root architecture (as mentioned above). Advances in imaging have been able to offset some of these hands-on analyses: high-resolution RGB imaging can differentiate between soil types facilitating soil type detection, which can improve mapping and hence conservation efforts (Potter and Weigand 2018).

New approaches that overcome the limitations of laboratory tests include thermal infrared imaging, which can be used to assess soil moisture distribution and hydraulic properties (Boulet et al. 2009) and inform land surface models (Kustas and Anderson 2009). Nearinfrared spectroscopy has been used for rapid and accurate identification of soil total nitrogen (TN), organic matter (OM), and $\mathrm{pH}$ levels in soil that can replace laboratory techniques (He et al. 2007; Ning et al. 2018). Similarly, hyperspectral imaging (HSI) can be used to accurately provide TN, OM, and organic content information in various soils (O'Rourke and Holden 2012) as well as fungal viability based on pixel spectra specific to browned, damaged, and undamaged tissue types (Taghizadeh et al. 2011). Because image processing of HSI is more challenging than that of RGB imaging, the two technologies can be used in tandem; for example, to optimize comprehensive analyses of soil and root systems in rhizoboxes (Bodner et al. 2017). The accuracy of both IR and HSI can be improved by applying extreme learning machine models, which were previously used to increase the accuracy of soil moisture and surface temperature measurements (Bai et al. 2015).

Because UAVs are scalable and programmable, we expect that drone usage in phytobiome research will move toward autonomous UAV fleets that can monitor extensive fields with an array of cheaper and more accurate sensors. We also expect aerial monitoring to be more closely coupled to robotics on the ground that could aid in conducting soil and plant analysis and deployment and maintenance of local sensor networks among various other tasks. Thus far, the development of robotics to measure soil characteristics has primarily focused on applications in environments that are difficult or unsafe to access. For instance, a robot was developed for measuring soil strength over depth, which is normally manually measured using a penetrometer, in unsafe zones (Cao et al. 2003). The Mars Phoenix Lander returned in situ measurements of Mars soil temperature, generated a topography map using imaging, and excavated soil samples for downstream testing (Arvidson et al. 2009).

\section{PLANT AND MICROBIAL CHEMISTRY}

Plant-microbiome signaling and metabolism rely on exchange of a large diversity of metabolites derived from microorganisms, plants, and the soil environment. Metabolomic methods enable direct characterization of these small molecules from soils and the various biological components. Given the large diversity of compounds that reside intra- and extracellularly in these systems, mass spectrometry (MS) coupled to chromatography such as liquid chromatography (LC)-MS and gas chromatography (GC)-MS have become primary methods for chemical analysis. Both techniques are well suited for identification and quantification of a wide range of molecules found in biological and environmental samples by coupling the physical separation of the compounds using LC with the separation and analysis of ions using MS mass (Jenkins et al. 2017). GC-MS and LC-MS are complementary in many ways. GC-MS typically has higher resolving power and produces richer fragmentation spectra, which makes it particularly well suited for identifying molecules such as small glycans that are often difficult to characterize by LC-MS. It is also well suited for volatile molecules and poorly ionizing molecules (e.g., hydrocarbons) that are often lost or not detected by LC-MS. LC-MS, on the other hand, is better suited for thermally labile compounds and is a technique of choice for analysis of novel compounds. Typically, these approaches are suitable for identification of several hundreds of metabolites based on spectral databases and authentic standards (Vinaixa et al. 2016). However, they are currently far from comprehensive, and improving metabolite identification is an important goal of metabolomics research.

A number of studies have used MS-based metabolomics to examine the chemical exchanges within phytobiomes; for example, the signaling molecules that direct the establishment of bacterial and mycorrhizal pathogens or symbionts with host plants. A number of metabolites have been identified, including sugars, amino acids, organic acids, phenolic compounds, and plant hormones, that are associated with beneficial interactions and are also implicated by single-strain and whole-community approaches (O'Banion et al. 2020). Exometabolite profiling (characterization of the extracellular metabolite pool) methods have been used to examine root exudates and their function in recruiting soil bacteria (Zhalnina et al. 2018a). O'Banion et al. (2020) have reviewed the function of the main chemical constituents of plant microbe signaling. Similarly, chemical imaging of solutes in soils has been reviewed (Santner et al. 2015). Although MS imaging (untargeted investigation into the spatial distribution of molecular species in a sample) is a powerful and promising technique (Buchberger et al. 2018), it is extremely difficult to identify organic components from complex environmental samples due to chemical complexity of these samples and the lack of physical separation of compounds prior to ionization. New developments in using ion mobility to separate ions within mass spectrometers have tremendous potential to overcome these limitations and enable direct analysis of metabolites from tissues and environmental samples (Spraggins et al. 2019).

\section{PLANT GENETICS}

It is well known that phytobiomes are affected by plant growth form and life history (e.g., forbs, shrubs, and trees; annuals versus 
perennials), plant community composition and habitat of origin, and even host plant species (Fitzpatrick et al. 2018). In fact, there is growing evidence of that intraspecific variability of plant hosts produces variability in phytobiomes (Singer et al. 2018; Wagner et al. 2016). Genetic differences within host species can affect microbe recruitment, community assembly, and, ultimately, the composition of phytobiomes. As such, the phytobiome can be considered an extended phenotype of the plant that is determined by host genetics, the environment, and their complex interaction. Here, the standard tools of quantitative genetics can be used to study the phytobiome. For example, family experimental designs or kinship-based mixed models can be used to partition variation in microbial abundance or composition into genetic and environmental components of variance for an entire assemblage of microbes associated with a particular plant compartment. This approach can provide insight into the host genetic architecture of the plant microbiome and, potentially, help to identify classes of microbes with close affinities for specific genotypes within a population.

A number of recent publications have documented genetic variation within plant species for aspects of the microbiome, including providing estimates of heritability for overall microbial community diversity and richness and for the abundance of specific microbial taxa based on counts derived from amplicon sequencing, for example (Peiffer et al. 2013; Rodríguez et al. 2020; Walters et al. 2018) The majority of such studies have focused on crop plants in agronomic settings and little is known about the heritability of microbes from more natural populations; one exception to this is the outdoor study of Bergelson et al. (2019). We imagine that some of these host genetic effects are related to available habitat for microbial establishment (e.g., the extent of fine root growth), to resources shared with microbes as root exudates, or from more complex immune responses in the plant. Incorporating host genetics in plant microbiome studies is promising because it will point to mechanisms leading to beneficial or deleterious plant-microbe interactions, as well as leverage the growing resources available in plant genomics.

In order to more efficiently develop and deploy improved plant varieties, it is valuable to identify the causal genes or genetic markers underlying agronomic traits (e.g., yield) and disease resistance (Visscher et al. 2017). In addition, there is a need to understand the plant genes that influence the composition and function of the microbiome to improve our understanding and in order to maximize plant productivity. Two methods are commonly used to identify genes or markers associated with quantitative traits: quantitative trait locus (QTL) mapping and genome-wide association studies (GWAS). Both approaches rely on genome-wide scans for statistical association between polymorphic genetic markers and quantitative variation in a measured phenotype. In the case of phytobiomes, the phenotype of interest could be a feature of the aggregate microbial community (e.g., community richness and evenness, principal coordinate analysis scores describing the microbial community) or an estimate of the relative abundance of a specific taxon (Beilsmith et al. 2019). A key distinction between these methods is that QTL mapping populations are derived from lines crosses and, therefore, represent experimentally structured populations, whereas GWAS focus on naturally occurring individuals. QTL mapping tends to have more power to detect true associations but reduced ability to localize effects in the genome because of limited recombination in a breeding population. In contrast, GWAS are frequently underpowered, given limited sample sizes, but can yield remarkably fine-scaled localization due to extensive historical recombination. It can also be much faster to establish a GWAS population than a QTL population because there is no need to create recombinant progeny through complex breeding designs across multiple generations. However, GWAS requires dense markers and reliable controls for population structure and, at best, yields correlative results rather than causal inference as achieved with QTLs. Because, in QTL studies, fewer alleles and markers are analyzed using a randomized genetic background, statistical analysis can yield causal relationships between alleles and traits (Lowry et al. 2015). Although both GWAS and QTL analyses establishing relationships between plant genetics and phenotypic traits are common, links between plant genetics and microbiome composition and function have been rare. The earliest studies utilizing this approach focused on plant-related microbial diseases (Bartoli and Roux 2017), including fungal, oomycete, and bacterial pathogens. More recently, studies utilizing the model plant Arabidopsis thaliana have been published that explore complete microbial communities based on $16 \mathrm{~S}$ rRNA gene amplicon sequencing. For example, Horton et al. (2014) identified host loci that influence fungal and bacterial colonization density on leaves across an $A$. thaliana population in the field and found that loci encoding defense and cell wall integrity affect bacterial and fungal community variation, whereas loci that influence the reproduction of viruses, trichome branching, and morphogenesis affect bacterial species richness. Similarly, Wallace et al. (2018) looked at the leaf microbial communities across maize lines and found that functions related to short-chain carbon metabolism, secretion, and nitrotoluene degradation primarily encoded by Methylobacteria spp. are heritable metabolic traits, and that few plant loci were found to be significantly associated.

These studies provide an exciting glimpse of the potential importance of host genetic variation in the phytobiome and give a clear path to the identification of candidate genes. Future studies will help to define the groups of microbes with strong host impacts, as well as identify new genetic and metabolic pathways important in plantmicrobe interactions. Although aggregate community metrics may be relatively straightforward to generate, they may be difficult to interpret and less meaningful than studies focused on individual microbial species. However, it is also unclear how to best define microbial taxa for counting-what inference can be made from amplicon sequence variants, traditionally defined operational taxonomic units, or gene content abundance derived from enrichment or metagenomic analyses? Finally, genome-wide studies carry a heavy multiple testing burden due to dense testing both across genomes and also across multiple taxa or phenotypes. Care will need to be taken to limit false positives and misleading inferences-methods developed for other "omics"-based quantitative genetic systems such as expression or metabolic QTL analyses may provide helpful directions as the field matures.

\section{FABRICATED ECOSYSTEMS}

In an effort to conduct plant microbiome research across biologically meaningful spatiotemporal scales and with increased control, a range of fabricated ecosystems are being developed. Experimental control and complexity are inversely related in plant microbiome research. At the most extreme, controlled laboratory experiments are often binary (one plant and one microbe), whereas field experiments feature real-world complexity that is difficult to replicate year by year. A new generation of experimental platforms of increasing complexity now allows for multifactorial insight, reproducibility, and increased statistical power.

The concept of controlled environments for exploring plant ecophysiology dates back to the late 1940s, when Frits Went developed a Phytotron at Caltech (Munns 2014), a "Climatron" in St. Louis, MO (https://www.missouribotanicalgarden.org/gardensgardening/our-garden/gardens-conservatories/conservatories/ 
climatron.aspx), and an ecophysiology lab at the Desert Research Institute, University of Nevada, Reno, which is now home to the recently developed EcoCELLs (https://www.dri.edu/labs/ecocells/). Went's work inspired the development of the EcoTron program at Centre National de la Recherche Scientifique, Montpellier, France (https://www.ecotron.cnrs.fr/macrocosms/), and the EcoTron at Imperial College London, United Kingdom (Fig. 2).

EcoTrons are large, fabricated ecosystems that consist of an aboveground dome of approximately $40 \mathrm{~m}^{3}$ and a belowground chamber that contains a lysimeter that can hold 2 to 12 tons of soil (up to $5-\mathrm{m}^{2}$ footprint and up to $2-\mathrm{m}$ soil depth). The canopy area is up to $2 \mathrm{~m}$ tall and allows work under natural light as well as under controlled or artificial light conditions. Both above- and belowground compartments are equipped with arrays of sensors and instrumentation for environmental control. Using the EcoTron, simulations of a wide range of environmental scenarios under realistic conditions can be performed, while measurements important for ecosystem processes such as atmospheric and soil gas composition, temperature, and $\mathrm{pH}$, among others, can be conducted. Studies in EcoTrons will be increasing in the near future and will provide unprecedented insights into ecosystem functioning; for example, Roscher et al. (2019) found that the functional composition of communities is key in explaining carbon assimilation in grasslands.
Mesocosms, which we call EcoPODs, are smaller versions of EcoTrons with higher experimental throughput that bridge laboratory and field studies (Eisenhauer and Türke 2018). Existing EcoPODs have a footprint of approximately $2.1 \mathrm{~m}^{2}$ and can be filled with up to $1.23 \mathrm{~m}^{3}$ of soil of $0.8 \mathrm{~m}$ in depth (UGT GmbH 2017). Using the EcoPOD lysimeter technology, intact soil monoliths can be retrieved from the field and studied under controlled conditions in the laboratory. The aboveground portion is approximately $1.5 \mathrm{~m}$ tall and, therefore, allows the study of a number of different plants in soil with macro- and microorganisms in the context of environmental changes. The contained nature of EcoPODs allows accurate mass balance calculations (e.g., tracking of greenhouse gases over time). EcoPODs allow precise conditioning of above- and belowground temperature and moisture and, therefore, can simulate seasonal changes and enable short- as well as long-term experiments. They are equipped with state-of-the-art sensor technology allowing in situ measurements of key environmental parameters, activities (fluxes) of organisms, and ecosystems at micrometer to meter scales. EcoPODs can be equipped with multi- and hyperspectral cameras that track plant biomass and physiological states. In conjunction with highly controlled physical and chemical conditions, researchers will be able to track the microbial activity within the system using a variety of genomic tools, including DNA or RNA shotgun metagenomics, proteomics, and metabolomics. This will facilitate tracking of

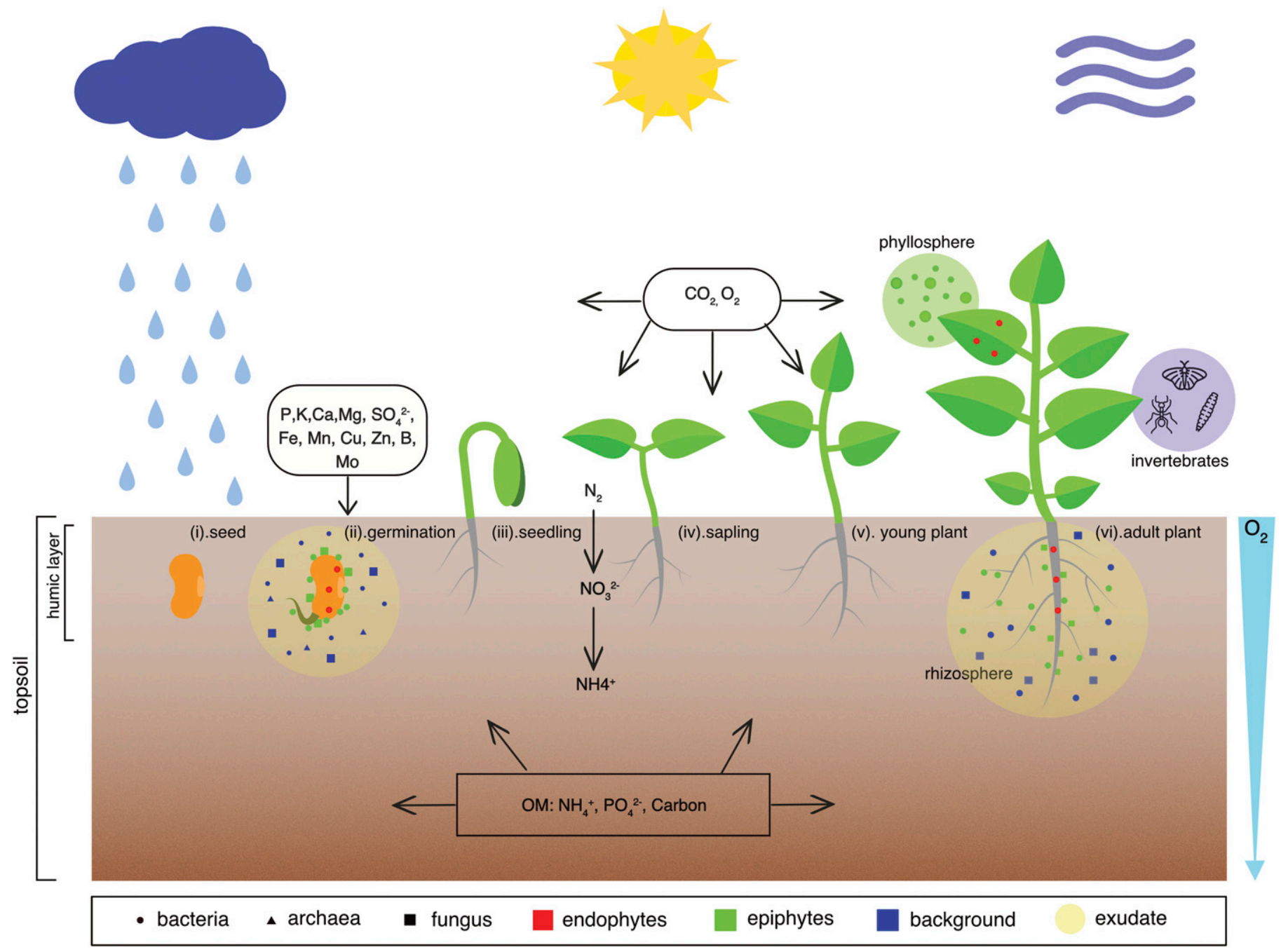

Fig. 1. Overview of abiotic and biotic factors affecting plant, microbiome, and overall soil health. 
microbial recruitment and activity across all life stages of the plant and can simulate seasonal changes. Broadly, this system can be used for fundamental research questions about biogeochemical cycles and the role of biodiversity in ecosystem processes, as well as applied studies that include biological or chemical components that require increased safety clearance and cannot be easily tested in the field. Because soil ecosystem and phytobiome experiments increasingly rely on in situ sensing over time, EcoPODs can also serve as a testbed for novel and improved sensing capabilities.
Complimenting approaches to develop more field-relevant laboratory growth systems are composed of one or more single plant chambers such as RootChips (Grossmann et al. 2011), GLO-ROOT (Rellán-Álvarez et al. 2015), EcoFAB (Zengler et al. 2019), and other systems that enable detailed characterization. For example, RootChips systems provide a high-throughput system for rhizosphere imaging, the GLO-ROOT systems enable direct imaging of root architecture within soils, and EcoFABs are "fabricated ecosystems" that are aimed at creating model

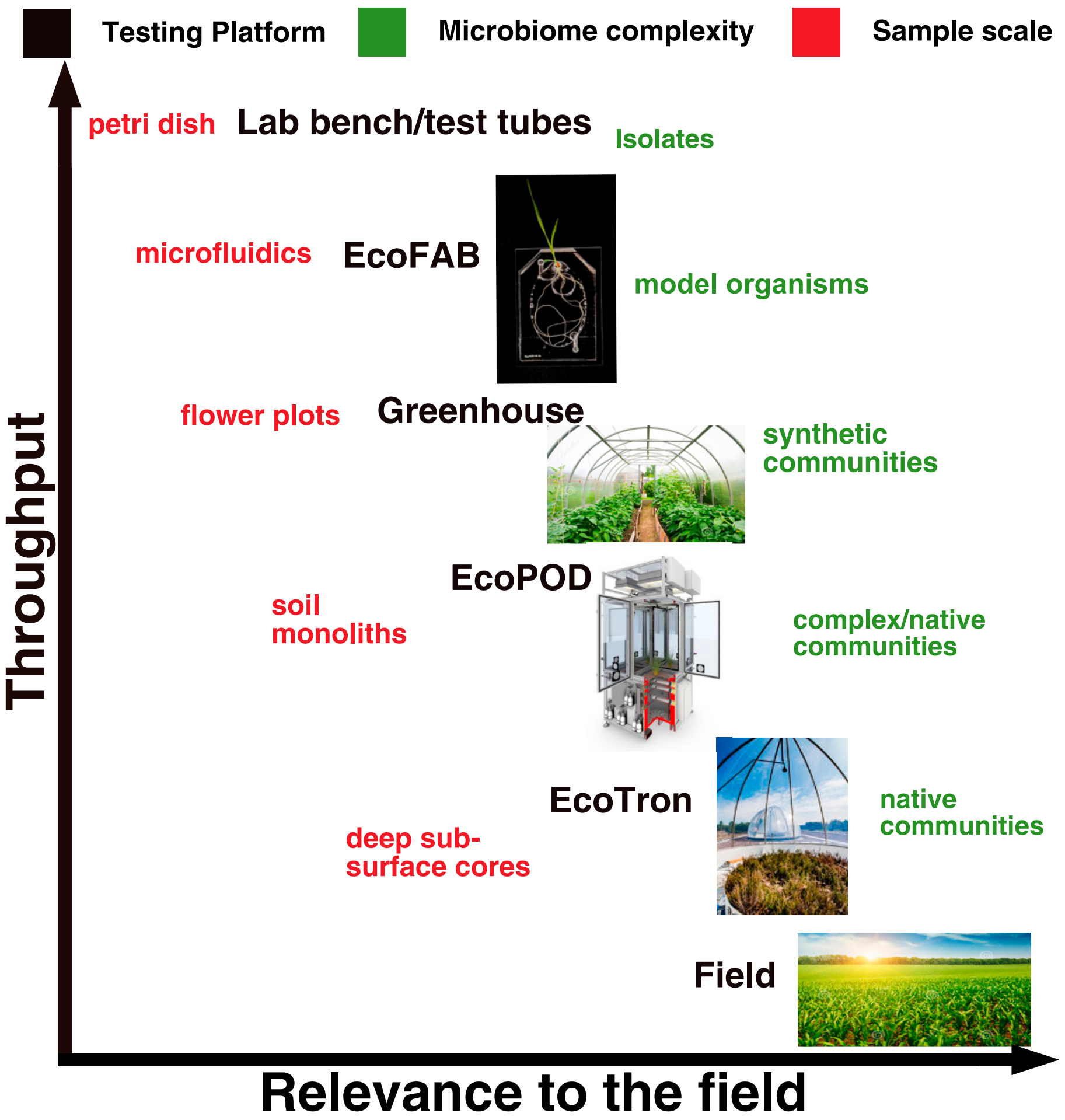

Fig. 2. Most commonly used experimental platforms for plant microbiome studies displayed along a scale of experimental throughput versus relevance to the field. Figure credit: EcoFAB: Dr. Lauren Jabusch; EcoTron: Dr. Sofie Thijs, Nathalie Beenaerts; and EcoPOD: Umwelt Geräte Technik, Germany. 
ecosystems on par with the model organisms used for genetic and biological studies.

EcoFABs comprise a chamber, biological and abiotic components (e.g., soil, plants, and microbes), and any measurement technologies (e.g., sensors or microfluidic sampling apparatus). EcoFABs allow real-time microscopy for high-resolution imaging of plant root architecture and are currently designed to provide sufficient materials for metabolomic, geochemical, and sequencebased analyses. They are made using widely accessible 3D printing technologies to fabricate controlled microbiome habitats that can be standardized and easily disseminated between labs (Sasse et al. 2019; Zhalnina et al. 2018b). This approach provides flexibility that enables scientists to add or change variables while monitoring microorganisms and their interaction with plants. Replicability in EcoFABs has recently been demonstrated in a ring trial across multiple laboratories (Sasse et al. 2019). EcoFABs are also envisioned to facilitate standardization of phytobiome research because construction materials are cheap and construction instructions are available (Gao et al. 2018).

Analogous to medical drug testing pipelines, which generally begin as high-throughput laboratory screens and are gradually scaled up to relevant mammal models and, finally, to human clinical trials, we envision phytobiome research studies to similarly follow a throughput versus relevance gradient from EcoFABs to EcoPODs and, finally, to field studies (Fig. 2). Although this suite of fabricated ecosystems is not aimed at simulating the real world, the enhanced control over abiotic and biotic factors in these experimental platforms enables plant root microbiome interaction studies that are not possible in field experiments because fields generally display greater complexity and unpredictability or do not allow for manipulations. Thus, use of fabricated ecosystems can reveal important correlations and causations of individual metabolic reactions as well as biogeochemical cycles. Challenges that have been encountered or are foreseeable include the relatively short experimental durations that can be executed in EcoFABs as well as EcoPODs due to the size limitations of the respective platforms and because of potential increases in parasite pressure as a result of air and water flow limitations. On the other hand, EcoTrons are not set up for quick turnover experiments and require expensive infrastructure to start and end experiments. Although insights obtained from greenhouse experiments have often not been replicable in the field, we expect that EcoFAB can serve as a reproducible system, in which microscopy and metabolomics can be applied to lowcomplexity microbiomes in the context of plant roots. Data obtained from individual microorganisms can inform microbially based biogeochemical models, as discussed below. We expect EcoPODs and EcoTrons to facilitate in situ sensing, climate manipulations, and deep soil monolith access. Links and extrapolations among fabricated ecosystems and the field can be achieved by generating and testing hypotheses across platform scales. For example, field observations may be tested under replicable conditions in EcoTron or EcoPOD and promising microbial candidates could be isolated and further studied in EcoFABs. A reverse workflow is also imaginable, where promising microbial isolates or plants resulting from EcoFAB experiments may be tested in EcoPOD or EcoTron before being potentially released into field experiments. Furthermore, extrapolations could be testable beforehand by taking advantage of archived datasets from sources such as long-term observatories, including Neon (https://www.neonscience.org/). Generally, challenges for extrapolating results of these fabricated ecosystems to realistic field conditions could be presented by the limited complexity in these laboratory systems; for example microbial isolates often perform predictably under laboratory conditions but may be inactivated by night temperatures or competitors.
There is still a number of unknown unknowns which may significantly affect plant performance, microbial community dynamics, and soil nutrient cycling, and which vary from ecosystem to ecosystem, hence resulting in a disconnect between studies conducted in the laboratory versus in the field. Other challenges are presented by natural climate variability in the field and the uncertainty in climate change predictions, which are significantly affected by socioeconomical drivers (Moss et al. 2010). Although laboratory experiments may be conducted based on historic field data or even in tandem with real-time field data measurements-for example, using sensor platforms coupled to edge computing (Beckman et al. 2016) - results may have limited applicability under future climate scenarios. However, this is also true for reproducibility of field experiments in general. Studying plant microbiome interactions and soil processes under defined conditions can assist in the identification and evaluation of such unknown unknowns which, in turn, will improve applicability of laboratory results to the field.

\section{SYNTHETIC COMMUNITIES}

Microbial communities found on healthy plants are incredibly taxonomically diverse and include bacteria, archaea, fungi, oomycetes, algae, protozoa, nematodes, and viruses (Bulgarelli et al. 2013; Kemen 2014; Lu and Conrad 2005; Turner et al. 2013). This microbial complexity makes it impossible to definitively establish causal relationships between plant and microbial genotypes and phenotypes as well as environmental factors. Instead, representative synthetic communities (SynComs) of defined complexity enable systematic bottom-up approaches in gnotobiotic systems under controlled and reproducible conditions to determine causal relationships (Lebeis et al. 2015).

In order to systematically test plant microbial community dynamics and functions in relation to the chemical composition of the surrounding environment, comprehensive strain collections representing the phylogenetic and functional diversity of the plant microbiota have been established thanks to the cultivability of an unexpectedly large fraction of the members of the plant microbiota (Müller et al. 2016). This high cultivability of plant-associated bacteria is likely based on low-complexity food webs, continuous substrate supply by the plant, and an essentially aerobic environment (Müller et al. 2016). In addition to cultivation and subsequent whole-genome analysis, screening SynComs of various complexity for interactions and metabolic activity in correlation with environmental parameters has been a bottleneck. Microfluidics tools such as massively parallel on-chip coalescence of microemulsions enable screening of 100,000 communities per day (Kehe et al. 2019). For example, bacterial isolates can be screened individually and in combinatory sets as SynComs for various useful properties, including plant-growth-promoting functions such as suppression of pathogens or degradation of harmful substrates, for their potential in biofuel production, or as environmental remediation agents. Such tools coupled with high-throughput DNA or RNA sequencing and long-read sequencing platforms including PacBio and Oxford Nanopore (Sevim et al. 2019), as well as metabolomics and various activity assays (Singer et al. 2019), now allow rapid profiling composition, function, and activity of SynComs as well as complex native microbial communities residing in soils and on plants.

\section{DATA INTEGRATION, MODELING, AND PREDICTION}

The quantity of data generated by the new technologies described above surpasses the capabilities of traditional analysis methods. Nevertheless, to gain insight, we need to integrate and fuse different 
data streams. To accomplish this, we must overcome the heterogeneous data types and lack of standards for data exchange. Ultimately, we need systems that can dynamically pull in diverse data from different devices and experimental modalities and intelligently interpret it using background knowledge in order to derive new hypotheses or make predictions such as being able to predict the consequences of specific environmental changes on plant health mediated by the microbiome.

Machine-learning (ML) methods and, in particular, deep learning (DL) have proven particularly useful for classification problems involving large datasets such as environmental data generated from technologies, including thermal sensing and LiDAR. Supervised ML techniques will learn to classify entities based on vectors of data characteristics, trained from prelabeled data. DL techniques involve the use of multilayer architecture neural networks (NNs). Different DL architectures can be applied to different problems. Convolution networks can be applied to image detection and recognition problems (for example, detecting and segmenting the anatomical components of a plant, and assessing its health), whereas recurrent architectures such as long short-term memory can be applied to time-series data. One of the challenges of phytobiome data are the paucity of sample data or lack of resolution in imaging and instrumentation. One DL architecture designed to address this is the generative adversarial network (GAN). A GAN can generate plausible synthetic data by utilizing two NNs that are trained together in an adversarial scenario-one network (the discriminator) attempts to distinguish real examples from fake ones, and the other (the generator) creates plausible example data to fool the first. Over time, both models improve, and the generated examples become more plausible, reflecting real-world characteristics of the domain without the need for explicit encoding of priors. In the context of phytobiome data, GAN could, for instance, help to synthesize and denoise imaging data (Yang et al. 2018). Although DL has seen tremendous gains and achieved much over the last decade, there are still a number of challenges. The input data must be in vector form, which is straightforward for sensor data; however, complex biological information must be embedded in a suitable fashion. NNs are famously inscrutable - they do not provide any explanation as to why they produce a particular result. This is particularly problematic in the face of adversarial attacks, in which the $\mathrm{NN}$ is deliberately fooled by fake data designed to elicit a misclassification. The burgeoning field of explainable artificial intelligence (Basu et al. 2018) attempts to use a variety of techniques to make DL decision making less of a black box process.

The field of DL and ML has seen a rapid advance in recent years but, in many cases, DL methods may not yield improvements over traditional methods. DL methods are best applied for complex multidimensional data such as imaging data or for predictions involving complex latent nonlinear mechanisms; for example, as found in ecosystem models. Some have successfully applied DL methods to modeling distinct ecosystem parameters such as soil temperature over a soil depth profile (Gagne et al. 2020), and processes such as ice-shelf melting as part of the Energy Exascale Earth System Model (Caldwell et al. 2019). DL methods will also gain importance in microbe-enabled soil biogeochemical models that aim to predict links between climate change, elevated $\mathrm{CO}_{2}$ concentrations, plant-microbe interactions, and soil nutrient cycling (Georgiou et al. 2017; Tang and Riley 2015; Treseder et al. 2011; Zhu et al. 2016). For example, the ecosys model (https:// github.com/jinyun1tang/ECOSYS) allows for the incorporation of microbially based models using traits such as growth rate (VieiraSilva and Rocha 2010), optimal temperature (Zeldovich et al. 2007), and resulting enzyme activity (Allison et al. 2010), as well as genome size. Microbial traits, which can be obtained from genomic data, help to identify and quantify trade-offs (Ferenci 2016). Trade-offs determine evolutionary adaptation constrains in bacterial properties and prevent ecological fitness in all environments and, therefore, are important parameters to include in ecosystem models.

Most data have to be cleaned, filtered, integrated, and processed before they can be utilized in ML or DL applications. This can be particularly challenging for plant-microbe-soil ecosystem data, which spans multiple scientific disciplines and technologies and results in vastly different data types with large ranges of data volume and temporal and spatial scales. The integration of these data types and standardization of analysis has been notoriously difficult, especially for data types that have been generated with rapidly evolving technologies such as nucleic acid sequencing. This difficulty in data integration combined with the incomplete collection of datasets still poses challenges for the development and application of current earth system modeling algorithms.

In order to compare between experiments, central and public data storage is fundamental. The use of standardized metadata combined with open controlled vocabularies or ontologies is crucial to being able to interoperate between different data types. The "findable, accessible, interoperable, reusable" (FAIR) data principles are aimed at improving the data ecosystem to allow researchers to better locate and integrate data. In the phytobiome sphere, the National Microbiome Data Collaborative (Wood-Charlson et al. 2020) is a new initiative to make microbiome data FAIR and aims to use standards such as the Environment Ontology to describe environmental characteristics of samples and the microbial ecosystems embedded within them. One of those limitations surrounding reference databases is the paucity of experimentally validated data that links microbial and plant metabolism, protein function, and DNA sequence. As an example, whereas microbial genes are assigned putative functions based on sequence homology, their actual activity may deviate from these annotations leading, to incorrect interpretations and predictions of ecosystem function (U.S. DOE 2019).

Furthermore, standardized analysis is critical and can be achieved by using centrally updated, state-of-the-art software tools. KBase, the U.S. Department of Energy Systems Biology Knowledgebase, has offered a public data storage and analysis dashboard that allows the generation of so-called narratives in which a dataset undergoes a string of analyses (Arkin et al. 2018). In addition, large-scale field datasets are increasingly taking advantage of supercomputer resources and ML algorithms that are required to filter noise and generate sensible interpretations from billions of data points.

\section{OUTLOOK}

The development of the abovementioned technologies and experimental platforms will improve our understanding of the plant-microbe-atmosphere-soil ecosystem at high spatial and temporal resolution. The combination and integrated use of the discussed tools will further provide opportunities for novel approaches to plant root microbiome research. An example of an integrated approach is the combination of UAVs equipped with advanced imaging capabilities to study QTL or GWAS populations growing in the field. This would streamline and scale current experimental procedures, so that new genetic markers for various above- and belowground phenotypic characteristics could be identified. These, in turn, could be correlated to microbiome community profiles in roots and leaves.

Another example is the combined usage of SynComs and single plants in EcoFabs for advanced root and microbe imaging resolved over space and time complemented with metabolite analysis, 
enabling systematic examination of the role of specific microbes and metabolites in modifying root architecture. This approach can help identify novel, specific microbial products that can be used to influence important plant traits known to affect field performance (e.g., root surface area). Microbial model systems can then be engineered to produce promising compounds for tests on plants in soil. Finally, we foresee EcoPODs and EcoTrons being used for time-series experiments that span several weeks and months, possibly years, in which high-throughput omics together with continuous environmental sensor measurements can provide indepth yet broad-scale datasets that can be used for training artificial intelligence algorithms related to biogeochemical cycling in relationship to climate.

Due to the many direct and indirect ties between local plantmicrobe-soil-ecosystem well-being and systems-wide ecological health, technological improvements in phytobiome research are directly translatable to improvements in climate change research. The abovementioned advances in instrumentation and methodology push precision agriculture and precision phytobiome research forward and allow for improved and more sustainable crop productivity under rapidly changing and increasingly extreme climatic conditions. These advances will have impacts in food and energy security and biosafety as well as environmental conservation and bioremediation.

\section{ACKNOWLEDGMENTS}

We thank N. Bouskill for discussion on modeling and V. Shah for assistance with Figure 1.

\section{LITERATURE CITED}

Alegria Terrazas, R., Giles, C., Paterson, E., Robertson-Albertyn, S., Cesco, S., Mimmo, T., Pii, Y., and Bulgarelli, D. 2016. Plant-microbiota interactions as a driver of the mineral turnover in the rhizosphere. Pages 1-67 in: Advances in Applied Microbiology, Vol. 95. S. Sariaslani and G. M. Gadd, eds. Academic Press, Elsevier, Cambridge, MA, U.S.A.

Allison, S. D., Wallenstein, M. D., and Bradford, M. A. 2010. Soil-carbon response to warming dependent on microbial physiology. Nat. Geosci. 3: 336-340.

Aravena, J. E., Berli, M., Menon, M., Ghezzehei, T. A., Mandava, A. K., Regentova, E. E., Pillai, N. S., Steude, J., Young, M. H., Nico, P. S., and Tyler, S. W. 2013. Synchrotron X-Ray microtomography-New means to quantify root induced changes of rhizosphere physical properties. Pages 39-67 in: Soil-Water-Root Processes: Advances in Tomography and Imaging. S. H. Anderson and J. W. Hopmans, eds. SSSA Special Publication 61. Soil Science Society of America, Inc., Madison, WI, U.S.A.

Arkin, A. P., Cottingham, R. W., Henry, C. S., Harris, N. L., Stevens, R. L., Maslov, S., Dehal, P., Ware, D., Perez, F., Canon, S., Sneddon, M. W., Henderson, M. L., Riehl, W. J., Murphy-Olson, D., Chan, S. Y., Kamimura, R. T., Kumari, S., Drake, M. M., Brettin, T. S., Glass, E. M., Chivian, D., Gunter, D., Weston, D. J., Allen, B. H., Baumohl, J., Best, A. A., Bowen, B., Brenner, S. E., Bun, C. C., Chandonia, J.-M., Chia, J.-M., Colasanti, R., Conrad, N., Davis, J. J., Davison, B. H., DeJongh, M., Devoid, S., Dietrich, E., Dubchak, I., Edirisinghe, J. N., Fang, G., Faria, J. P., Frybarger, P. M., Gerlach, W., Gerstein, M., Greiner, A., Gurtowski, J., Haun, H. L., He, F., Jain, R., Joachimiak, M. P., Keegan, K. P., Kondo, S., Kumar, V., Land, M. L., Meyer, F., Mills, M., Novichkov, P. S., Oh, T., Olsen, G. J., Olson, R., Parrello, B., Pasternak, S., Pearson, E., Poon, S. S., Price, G. A.,

Ramakrishnan, S., Ranjan, P., Ronald, P. C., Schatz, M. C., Seaver, S. M. D., Shukla, M., Sutormin, R. A., Syed, M. H., Thomason, J., Tintle, N. L., Wang, D., Xia, F., Yoo, H., Yoo, S., and Yu, D. 2018. KBase: The United States Department of Energy Systems Biology Knowledgebase. Nat. Biotechnol. 36:566-569.

Arvidson, R. E., Bonitz, R. G., Robinson, M. L., Carsten, J. L., Volpe, R. A., Trebi-Ollennu, A., Mellon, M. T., Chu, P. C., Davis, K. R., Wilson, J. J., Shaw, A. S., Greenberger, R. N., Siebach, K. L., Stein, T. C., Cull, S. C., Goetz, W., Morris, R. V., Ming, D. W., Keller, H. U., Lemmon, M. T.,
Sizemore, H. G., and Mehta, M. 2009. Results from the Mars Phoenix Lander robotic arm experiment. J. Geophys. Res. Planets 114:E00E02.

Bai, Y., Wong, M. S., Shi, W.-Z., Wu, L.-X., and Qin, K. 2015. Advancing of land surface temperature retrieval using extreme learning machine and spatiotemporal adaptive data fusion algorithm. Remote Sens. 7:4424-4441.

Bartoli, C., and Roux, F. 2017. Genome-wide association studies in plant pathosystems: Toward an ecological genomics approach. Front. Plant Sci. 8:763.

Basu, S., Kumbier, K., Brown, J. B., and Yu, B. 2018. Iterative random forests to discover predictive and stable high-order interactions. Proc. Natl. Acad. Sci. U.S.A. 115:1943-1948.

Beckman, P., Sankaran, R., Catlett, C., Ferrier, N., Jacob, R., and Papka, M. 2016. Pages 1-3 in: Waggle: An open sensor platform for edge computing. Pages 1-3 in: 2016 IEEE SENSORS. IEEE, Orlando, FL, U.S.A.

Beilsmith, K., Thoen, M. P. M., Brachi, B., Gloss, A. D., Khan, M. H., and Bergelson, J. 2019. Genome-wide association studies on the phyllosphere microbiome: Embracing complexity in host-microbe interactions. Plant J. 97: 164-181.

Bendig, J., Bolten, A., Bennertz, S., Broscheit, J., Eichfuss, S., and Bareth, G. 2014. Estimating biomass of barley using crop surface models (CSMs) derived from UAV-based RGB imaging. Remote Sens. 6:10395-10412.

Bergelson, J., Mittelstrass, J., and Horton, M. W. 2019. Characterizing both bacteria and fungi improves understanding of the Arabidopsis root microbiome. Sci. Rep. 9:24

Bodner, G., Alsalem, M., Nakhforoosh, A., Arnold, T., and Leitner, D. 2017. RGB and spectral root imaging for plant phenotyping and physiological research: Experimental setup and imaging protocols. J. Vis. Exp. 126:e56251.

Boulet, G., Mougenot, B., and Abdelouahab, T. B. 2009. An evaporation test based on thermal infra red remote-sensing to select appropriate soil hydraulic properties. J. Hydrol. (Amsterdam, Neth.) 376:589-598.

Brodersen, C. R., McElrone, A. J., Choat, B., Lee, E. F., Shackel, K. A., and Matthews, M. A. 2013. In vivo visualizations of drought-induced embolism spread in Vitis vinifera. Plant Physiol. 161:1820-1829.

Buchberger, A. R., DeLaney, K., Johnson, J., and Li, L. 2018. Mass spectrometry imaging: A review of emerging advancements and future insights. Anal. Chem. 90:240-265.

Bulgarelli, D., Schlaeppi, K., Spaepen, S., van Themaat, E. V. L., and SchulzeLefert, P. 2013. Structure and functions of the bacterial microbiota of plants. Annu. Rev. Plant Biol. 64:807-838.

Caldwell, P. M., Mametjanov, A., Tang, Q., Roekel, L. P. V., Golaz, J.-C., Lin, W., Bader, D. C., Keen, N. D., Feng, Y., Jacob, R., Maltrud, M. E., Roberts, A. F., Taylor, M. A., Veneziani, M., Wang, H., Wolfe, J. D., Balaguru, K., Cameron-Smith, P., Dong, L., Klein, S. A., Leung, R., Li, H.-Y., Li, Q., Liu, X., Neale, R. B., Pinheiro, M., Qian, Y., Ullrich, P. A., Xie, S., Yang, Y., Zhang, Y., Zhang, K., and Zhou, T. 2019. The DOE E3SM coupled model version 1: Description and results at high resolution. J. Adv. Model. Earth Syst. 11:4095-4146.

Cao, P. M., Hall, E. L., and Zhang, E. 2003. Soil sampling sensor system on a mobile robot. Pages 304-310 in: Proc. Vol. 5267. Intelligent Robots and Computer Vision XXI: Algorithms, Techniques, and Active Vision. Photonics Technologies for Robotics, Automation, and Manufacturing, Providence, RI, U.S.A.

Carrión, V. J., Perez-Jaramillo, J., Cordovez, V., Tracanna, V., de Hollander, M., Ruiz-Buck, D., Mendes, L. W., van Ijcken, W. F. J., Gomez-Exposito, R., Elsayed, S. S., Mohanraju, P., Arifah, A., van der Oost, J., Paulson, J. N., Mendes, R., van Wezel, G., Medema, M. H., and Raaijmakers, J. M. 2019. Pathogen-induced activation of disease-suppressive functions in the endophytic root microbiome. Science 366:606-612.

Cochard, H., Delzon, S., and Badel, E. 2015. X-ray microtomography (microCT): A reference technology for high-resolution quantification of xylem embolism in trees. Plant Cell Environ. 38:201-206.

Edwards, J. A., Santos-Medellín, C. M., Liechty, Z. S., Nguyen, B., Lurie, E., Eason, S., Phillips, G., and Sundaresan, V. 2018. Compositional shifts in rootassociated bacterial and archaeal microbiota track the plant life cycle in fieldgrown rice. PLoS Biol. 16:e2003862.

Eisenhauer, N., and Türke, M. 2018. From climate chambers to biodiversity chambers. Front. Ecol. Environ. 16:136-137.

Ferenci, T. 2016. Trade-off mechanisms shaping the diversity of bacteria. Trends Microbiol. 24:209-223.

Fierer, N. 2017. Embracing the unknown: Disentangling the complexities of the soil microbiome. Nat. Rev. Microbiol. 15:579-590.

Fitzpatrick, C. R., Copeland, J., Wang, P. W., Guttman, D. S., Kotanen, P. M., and Johnson, M. T. J. 2018. Assembly and ecological function of the root microbiome across angiosperm plant species. Proc. Natl. Acad. Sci. U.S.A. 115:E1157-E1165. 
Gagne, D. J., Christensen, H. M., Subramanian, A. C., and Monahan, A. H. 2020. Machine learning for stochastic parameterization: Generative adversarial networks in the Lorenz '96 model. J. Adv. Model. Earth Syst. 12: e2019MS001896.

Gamalero, E., Trotta, A., Massa, N., Copetta, A., Martinotti, M. G., and Berta, G. 2004. Impact of two fluorescent pseudomonads and an arbuscular mycorrhizal fungus on tomato plant growth, root architecture and $\mathrm{P}$ acquisition. Mycorrhiza 14:185-192.

Gao, J., Sasse, J., Lewald, K. M., Zhalnina, K., Cornmesser, L. T., Duncombe, T. A., Yoshikunio, Y., Vogel, J. P., Firestone, M. K., and Northen, T. R. 2018. Ecosystem fabrication (EcoFAB) protocols for the construction of laboratory ecosystems designed to study plant-microbe interactions. J. Vis. Exp. 134: e57170.

Georgiou, K., Abramoff, R. Z., Harte, J., Riley, W. J., and Torn, M. S. 2017. Microbial community-level regulation explains soil carbon responses to longterm litter manipulations. Nat. Commun. 8:1223.

Glaeser, S. P., Imani, J., Alabid, I., Guo, H., Kumar, N., Kämpfer, P., Hardt, M., Blom, J., Goesmann, A., Rothballer, M., Hartmann, A., and Kogel, K.-H. 2016. Non-pathogenic Rhizobium radiobacter F4 deploys plant beneficial activity independent of its host Piriformospora indica. ISME J. 10:871-884.

Grossmann, G., Guo, W.-J., Ehrhardt, D. W., Frommer, W. B., Sit, R. V., Quake, S. R., and Meier, M. 2011. The RootChip: An integrated microfluidic chip for plant science. Plant Cell 23:4234-4240.

He, Y., Huang, M., García, A., Hernández, A., and Song, H. 2007. Prediction of soil macronutrients content using near-infrared spectroscopy. Comput. Electron. Agric. 58:144-153.

Horton, M. W., Bodenhausen, N., Beilsmith, K., Meng, D., Muegge, B. D., Subramanian, S., Vetter, M. M., Vilhjálmsson, B. J., Nordborg, M., Gordon, J. I., and Bergelson, J. 2014. Genome-wide association study of Arabidopsis thaliana leaf microbial community. Nat. Commun. 5:5320.

Huuskonen, J., and Oksanen, T. 2018. Soil sampling with drones and augmented reality in precision agriculture. Comput. Electron. Agric. 154:25-35.

Iost Filho, F. H., Heldens, W. B., Kong, Z., and de Lange, E. S. 2020. Drones: Innovative technology for use in precision pest management. J. Econ. Entomol. 113:1-25.

Jenkins, S., Swenson, T. L., Lau, R., Rocha, A. M., Aaring, A., Hazen, T. C., Chakraborty, R., and Northen, T. R. 2017. Construction of viable soil defined media using quantitative metabolomics analysis of soil metabolites. Front. Microbiol. 8:2618.

Kehe, J., Kulesa, A., Ortiz, A., Ackerman, C. M., Thakku, S. G., Sellers, D., Kuehn, S., Gore, J., Friedman, J., and Blainey, P. C. 2019. Massively parallel screening of synthetic microbial communities. Proc. Natl. Acad. Sci. U.S.A. 116:12804-12809.

Kemen, E. 2014. Microbe-microbe interactions determine oomycete and fungal host colonization. Curr. Opin. Plant Biol. 20:75-81.

Koebernick, N., Daly, K. R., Keyes, S. D., George, T. S., Brown, L. K., Raffan, A., Cooper, L. J., Naveed, M., Bengough, A. G., Sinclair, I., Hallett, P. D., and Roose, T. 2017. High-resolution synchrotron imaging shows that root hairs influence rhizosphere soil structure formation. New Phytol. 216:124-135.

Kustas, W., and Anderson, M. 2009. Advances in thermal infrared remote sensing for land surface modeling. Agric. For. Meteorol. 149:2071-2081.

Lebeis, S. L., Paredes, S. H., Lundberg, D. S., Breakfield, N., Gehring, J., McDonald, M., Malfatti, S., del Rio, T. G., Jones, C. D., Tringe, S. G., and Dangl, J. L. 2015. Salicylic acid modulates colonization of the root microbiome by specific bacterial taxa. Science 349:860-864.

Liebisch, F., Kirchgessner, N., Schneider, D., Walter, A., and Hund, A. 2015. Remote, aerial phenotyping of maize traits with a mobile multi-sensor approach. Plant Methods 11:9.

Lin, E., and Alessio, A. 2009. What are the basic concepts of temporal, contrast, and spatial resolution in cardiac CT? J. Cardiovasc. Comput. Tomogr. 3: 403-408.

Lowry, D. B., Taylor, S. H., Bonnette, J., Aspinwall, M. J., Asmus, A. L., Keitt, T. H., Tobias, C M., and Juenger, T. E. 2015. QTLs for biomass and developmental traits in switchgrass (Panicum virgatum). BioEnergy Res. 8:1856-1867.

Lu, Y., and Conrad, R. 2005. In situ stable isotope probing of methanogenic archaea in the rice rhizosphere. Science 309:1088-1090.

Lucas, M. S., Guenthert, M., Gasser, P., Lucas, F., and Wepf, R. 2014. Correlative 3D imaging: CLSM and FIB-SEM tomography using highpressure frozen, freeze-substituted biological samples. Pages 593-616 in: Electron Microscopy. J. Kuo, ed. Methods in Molecular Biology (Methods and Protocols), Vol. 1117. Humana Press, Totowa, NJ, U.S.A.

Lundberg, D. S., Lebeis, S. L., Paredes, S. H., Yourstone, S., Gehring, J., Malfatti, S., Tremblay, J., Engelbrektson, A., Kunin, V., del Rio, T. G., Edgar, R. C., Eickhorst, T., Ley, R. E., Hugenholtz, P., Tringe, S. G., and Dangl, J. L.
2012. Defining the core Arabidopsis thaliana root microbiome. Nature 488: 86-90.

Mahlein, A.-K. 2016. Plant disease detection by imaging sensors-Parallels and specific demands for precision agriculture and plant phenotyping. Plant Dis. 100:241-251.

Mantelin, S., Desbrosses, G., Larcher, M., Tranbarger, T. J., Cleyet-Marel, J.-C., and Touraine, B. 2006. Nitrate-dependent control of root architecture and N nutrition are altered by a plant growth-promoting Phyllobacterium sp. Planta 223:591-603.

Mattupalli, C., Seethepalli, A., York, L. M., and Young, C. A. 2019. Digital imaging to evaluate root system architectural changes associated with soil biotic factors. Phytobiomes J. 3:102-111.

Metzner, R., Eggert, A., van Dusschoten, D., Pflugfelder, D., Gerth, S., Schurr, U., Uhlmann, N., and Jahnke, S. 2015. Direct comparison of MRI and X-ray CT technologies for 3D imaging of root systems in soil: Potential and challenges for root trait quantification. Plant Methods 11:17.

Milien, M., Renault-Spilmont, A.-S., Cookson, S. J., Sarrazin, A., and Verdeil, J.-L. 2012. Visualization of the 3D structure of the graft union of grapevine using X-ray tomography. Sci. Hortic. (Amsterdam) 144:130-140.

Minervini, M., Scharr, H., and Tsaftaris, S. A. 2015. Image analysis: The new bottleneck in plant phenotyping [Applications Corner]. IEEE Signal Process. Mag. 32:126-131.

Moss, R. H., Edmonds, J. A., Hibbard, K. A., Manning, M. R., Rose, S. K., van Vuuren, D. P., Carter, T. R., Emori, S., Kainuma, M., Kram, T., Meehl, G. A., Mitchell, J. F. B., Nakicenovic, N., Riahi, K., Smith, S. J., Stouffer, R. J., Thomson, A. M., Weyant, J. P., and Wilbanks, T. J. 2010. The next generation of scenarios for climate change research and assessment. Nature 463:747-756.

Müller, D. B., Vogel, C., Bai, Y., and Vorholt, J. A. 2016. The plant microbiota: Systems-level insights and perspectives. Annu. Rev. Genet. 50:211-234.

Munns, D. P. D. 2014. "The awe in which biologists hold physicists": Frits Went's first phytotron at Caltech, and an experimental definition of the biological environment. Hist. Philos. Life Sci. 36:209-231.

Ning, J., Sheng, M., Yi, X., Wang, Y., Hou, Z., Zhang, Z., and Gu, X. 2018. Rapid evaluation of soil fertility in tea plantation based on near-infrared spectroscopy. Spectrosc. Lett. 51:463-471.

O'Banion, B. S., O'Neal, L., Alexandre, G., and Lebeis, S. L. 2020. Bridging the gap between single-strain and community-level plant-microbe chemical interactions. Mol. Plant-Microbe Interact. 33:124-134.

O'Rourke, S. M., and Holden, N. M. 2012. Determination of soil organic matter and carbon fractions in forest top soils using spectral data acquired from visible-near infrared hyperspectral images. Soil Sci. Soc. Am. J. 76:586-596.

Peiffer, J. A., Spor, A., Koren, O., Jin, Z., Tringe, S. G., Dangl, J. L., Buckler, E. S., and Ley, R. E. 2013. Diversity and heritability of the maize rhizosphere microbiome under field conditions. Proc. Natl. Acad. Sci. U.S.A. 110: 6548-6553.

Potter, C., and Weigand, J. 2018. Imaging analysis of biological soil crusts to understand surface heating properties in the Mojave Desert of California. Catena 170:1-9.

Rellán-Álvarez, R., Lobet, G., Lindner, H., Pradier, P.-L., Sebastian, J., Yee, M.C., Geng, Y., Trontin, C., LaRue, T., Schrager-Lavelle, A., Haney, C. A., Nieu, R., Maloof, J., Vogel, J. P., and Dinneny, J. R. 2015. GLO-Roots: An imaging platform enabling multidimensional characterization of soil-grown root systems. eLife 4:e07597.

Rodríguez, C. E., Antonielli, L., Mitter, B., Trognitz, F., and Sessitsch, A. 2020. Heritability and Functional Importance of the Setaria viridis bacterial seed microbiome. Phytobiomes J. 4:40-52.

Roscher, C., Karlowsky, S., Milcu, A., Gessler, A., Bachmann, D., Jesch, A., Lange, M., Mellado-Vázquez, P., Strecker, T., Landais, D., Ravel, O., Buchmann, N., Roy, J., and Gleixner, G. 2019. Functional composition has stronger impact than species richness on carbon gain and allocation in experimental grasslands. PLoS One 14:e0204715.

Saleem, M., Law, A. D., Sahib, M. R., Pervaiz, Z. H., and Zhang, Q. 2018. Impact of root system architecture on rhizosphere and root microbiome. Rhizosphere 6:47-51.

Santner, J., Larsen, M., Kreuzeder, A., and Glud, R. N. 2015. Two decades of chemical imaging of solutes in sediments and soils-A review. Anal. Chim. Acta 878:9-42.

Sasse, J., Kant, J., Cole, B. J., Klein, A. P., Arsova, B., Schlaepfer, P., Gao, J., Lewald, K., Zhalnina, K., Kosina, S., Bowen, B. P., Treen, D., Vogel, J., Visel, A., Watt, M., Dangl, J. L., and Northen, T. R. 2019. Multilab EcoFAB study shows highly reproducible physiology and depletion of soil metabolites by a model grass. New Phytol. 222:1149-1160.

Sevim, V., Lee, J., Egan, R., Clum, A., Hundley, H., Lee, J., Everroad, R. C., Detweiler, A. M., Bebout, B. M., Pett-Ridge, J., Göker, M., Murray, A. E., 
Lindemann, S. R., Klenk, H.-P., O’Malley, R., Zane, M., Cheng, J.-F., Copeland, A., Daum, C., Singer, E., and Woyke, T. 2019. Shotgun metagenome data of a defined mock community using Oxford Nanopore, PacBio and Illumina technologies. Sci. Data 6:285.

Singer, E., Bonnette, J., Kenaley, S., Woyke, T., and Juenger, T. E. 2019. Plant compartment and genetic variation drive microbiome composition in switchgrass roots. Environ. Microbiol. Rep. 11:185-195.

Spraggins, J. M., Djambazova, K. V., Rivera, E. S., Migas, L. G., Neumann, E. K., Fuetterer, A., Suetering, J., Goedecke, N., Ly, A., Van de Plas, R., and Caprioli, R. M. 2019. High-performance molecular imaging with MALDI trapped ion-mobility time-of-flight (timsTOF) mass spectrometry. Anal. Chem. 91:14552-14560.

Taghizadeh, M., Gowen, A. A., and O'Donnell, C. P. 2011. The potential of visible-near infrared hyperspectral imaging to discriminate between casing soil, enzymatic browning and undamaged tissue on mushroom (Agaricus bisporus) surfaces. Comput. Electron. Agric. 77:74-80.

Tang, J., and Riley, W. J. 2015. Weaker soil carbon-climate feedbacks resulting from microbial and abiotic interactions. Nat. Clim. Change 5:56-60.

Torres-Ruiz, J. M., Jansen, S., Choat, B., McElrone, A. J., Cochard, H., Brodribb, T. J., Badel, E., Burlett, R., Bouche, P. S., Brodersen, C. R., Li, S., Morris, H., and Delzon, S. 2015. Direct X-Ray microtomography observation confirms the induction of embolism upon xylem cutting under tension. Plant Physiol. 167:40-43.

Treseder, K. K., Balser, T. C., Bradford, M. A., Brodie, E. L., Dubinsky, E. A., Eviner, V. T., Hofmockel, K. S., Lennon, J. T., Levine, U. Y., MacGregor, B. J., Pett-Ridge, J., and Waldrop, M. P. 2011. Integrating microbial ecology into ecosystem models: Challenges and priorities. Biogeochemistry 109:7-18.

Turner, T. R., Ramakrishnan, K., Walshaw, J., Heavens, D., Alston, M., Swarbreck, D., Osbourn, A., Grant, A., and Poole, P. S. 2013. Comparative metatranscriptomics reveals kingdom level changes in the rhizosphere microbiome of plants. ISME J. 7:2248-2258.

UGT GmbH. 2017. EcoUnit (Ecotrons). UGT Umwelt Geräte Technik. https:// www.ugt-online.de/en/products/lysimeter-technology/ecotrons/ecounit/

U.S. DOE. 2019. Breaking the Bottleneck of Genomes. Understanding Gene Function Across Taxa, Workshop Report DOE/SC-0199. United States Department of Energy, Office of Science, Bethesda, MD, U.S.A. https:// genomicscience.energy.gov/genefunction/

van Dusschoten, D., Metzner, R., Kochs, J., Postma, J. A., Pflugfelder, D., Bühler, J., Schurr, U., and Jahnke, S. 2016. Quantitative 3D analysis of plant roots growing in soil using magnetic resonance imaging. Plant Physiol. 170:1176-1188.

Vieira-Silva, S., and Rocha, E. P. C. 2010. The systemic imprint of growth and its uses in ecological (meta)genomics. PLoS Genet. 6:e1000808.

Vinaixa, M., Schymanski, E. L., Neumann, S., Navarro, M., Salek, R. M., and Yanes, O. 2016. Mass spectral databases for LC/MS- and GC/MS-based metabolomics: State of the field and future prospects. TrAC Trends Anal. Chem. 78:23-35.

Virlet, N., Lebourgeois, V., Martinez, S., Labbe, S., Costes, E., and Regnard, J. L. 2015. Phenotyping the response of an apple tree hybrid population to soil water constraint under field conditions: New insights brought by high resolution imaging. Acta Hortic. 1099:879-886.

Visscher, P. M., Wray, N. R., Zhang, Q., Sklar, P., McCarthy, M. I., Brown, M. A., and Yang, J. 2017. 10 Years of GWAS discovery: Biology, function, and translation. Am. J. Hum. Genet. 101:5-22.
Voltolini, M., Taş, N., Wang, S., Brodie, E. L., and Ajo-Franklin, J. B. 2017. Quantitative characterization of soil micro-aggregates: New opportunities from sub-micron resolution synchrotron X-ray microtomography. Geoderma 305:382-393.

Wagner, M. R., Lundberg, D. S., del Rio, T. G., Tringe, S. G., Dangl, J. L., and Mitchell-Olds, T. 2016. Host genotype and age shape the leaf and root microbiomes of a wild perennial plant. Nat. Commun. 7:12151.

Wallace, J. G., Kremling, K. A., Kovar, L. L., and Buckler, E. S. 2018. Quantitative genetics of the maize leaf microbiome. Phytobiomes J. 2: 208-224.

Walters, W. A., Jin, Z., Youngblut, N., Wallace, J. G., Sutter, J., Zhang, W., González-Peña, A., Peiffer, J., Koren, O., Shi, Q., Knight, R., del Rio, T. G., Tringe, S. G., Buckler, E. S., Dangl, J. L., and Ley, R. E. 2018. Large-scale replicated field study of maize rhizosphere identifies heritable microbes. Proc. Natl. Acad. Sci. U.S.A. 115:7368-7373.

Wei, X., Zhu, Z., Wei, L., Wu, J., and Ge, T. 2019. Biogeochemical cycles of key elements in the paddy-rice rhizosphere: Microbial mechanisms and coupling processes. Rhizosphere 10:100145.

Wood-Charlson, E. M., Anubhav, Auberry, D., Blanco, H., Borkum, M. I., Corilo, Y. E., Davenport, K. W., Deshpande, S., Devarakonda, R., Drake, M., Duncan, W. D., Flynn, M. C., Hays, D., Hu, B., Huntemann, M., Li, P.-E., Lipton, M., Lo, C.-C., Millard, D., Miller, K., Piehowski, P. D., Purvine, S., Reddy, T. B. K., Shakya, M., Sundaramurthi, J. C., Vangay, P., Wei, Y., Wilson, B. E., Canon, S., Chain, P. S. G., Fagnan, K., Martin, S., McCue, L. A., Mungall, C. J., Mouncey, N. J., Maxon, M. E., and Eloe-Fadrosh, E. A. 2020. The National Microbiome Data Collaborative: Enabling microbiome science. Nat. Rev. Microbiol. 18:313-314.

Yang, Q., Yan, P., Zhang, Y., Yu, H., Shi, Y., Mou, X., Kalra, M. K., Zhang, Y., Sun, L., and Wang, G. 2018. Low-dose CT image denoising using a generative adversarial network with Wasserstein distance and perceptual loss. IEEE Trans. Med. Imaging 37:1348-1357.

Zeldovich, K. B., Berezovsky, I. N., and Shakhnovich, E. I. 2007. Protein and DNA sequence determinants of thermophilic adaptation. PLOS Comput. Biol. 3:e5.

Zengler, K., Hofmockel, K., Baliga, N. S., Behie, S. W., Bernstein, H. C., Brown, J. B., Dinneny, J. R., Floge, S. A., Forry, S. P., Hess, M., Jackson, S. A., Jansson, C., Lindemann, S. R., Pett-Ridge, J., Maranas, C., Venturelli, O. S., Wallenstein, M. D., Shank, E. A., and Northen, T. R. 2019. EcoFABs: Advancing microbiome science through standardized fabricated ecosystems. Nat. Methods 16:567-571.

Zhalnina, K., Louie, K., Hao, Z., Mansoori, N., Nunes da Rocha, U., Shi, S., Cho, H., Karaoz, U., Loqué, D., Bowen, B. P., Firestone, M. K., Northen, T. R., and Brodie, E. L. 2018a. Dynamic root exudate chemistry and microbial substrate preferences drive patterns in rhizosphere microbial community assembly. Nat. Microbiol. 3:470-480.

Zhalnina, K., Zengler, K., Newman, D., and Northen, T. R. 2018b. Need for laboratory ecosystems to unravel the structures and functions of soil microbial communities mediated by chemistry. mBio 9:e01175-18.

Zhu, Q., Riley, W. J., Tang, J., and Koven, C. D. 2016. Multiple soil nutrient competition between plants, microbes, and mineral surfaces: Model development, parameterization, and example applications in several tropical forests. Biogeosciences 13:341-363. 